\title{
THE MAGNETIC PROPERTIES OF CERTAIN Pt-Co AND Pd-Co ALLOYS
}

\author{
By F. Woodbridge Constant*
}

Norman Bridge Laboratory, Pasadena, California

(Received September 11, 1930)

Abstract

\begin{abstract}
If ferromagnetism is due to interaction between the electrons of neighboring ferromagnetic atoms, the effect on the magnetic properties of gradually separating these atoms is of interest. For this reason alloys of the Pt-Co and Pd-Co series were investigated. As the alloys were solid solutions, for small cobalt content the cobalt atoms could be regarded as partly isolated by the non-ferromagnetic atoms. The magnetic properties of these alloys showed a lowering of the Curie point and a decrease in the remanent magnetization and coercive force with decrease in the percentage of cobalt in accord with theory. An observed increase in the magnetization per cobalt atom is discussed along with recent theories of ferromagnetism.
\end{abstract}

\section{INTRODUCTION}

$T_{\text {tiso }}^{H}$ HE explanation of ferromagnetism has presented much greater difficulties than that of either diamagnetism or paramagnetism. X-ray experiments on ferromagnetic crystals have shown that the elementary magnet cannot be larger than the atom, and the most recent work of Barnett ${ }^{1}$ on the gyromagnetic effect indicates that ferromagnetism can be attributed chiefly to the spinning electrons. But in spite of the supposed simple character of the elementary magnet experiments have shed little light on its behavior in ferromagnetic substances.

The point from which to attack the problem of magnetism most directly would seem to be the Gerlach and Stern ${ }^{2}$ experiments, in which the magnetic moment and behavior of an individual atom is measured. The sodium and cobalt atoms have been found to possess magnetic moments of one and six Bohr magnetons respectively, but in the solid state sodium is only paramagnetic while the magnetic moment per cobalt atom, computed for saturation magnetization, is about 1.7 magnetons. However, in the gaseous state of an atomic beam neither element can be classed as ferromagnetic. The fundamental question is what special mechanism in the solid state makes cobalt ferromagnetic and sodium not. Ferromagnetism arises only when certain atoms, (notably $\mathrm{Fe}, \mathrm{Ni}$ or $\mathrm{Co}$ ), are brought into the close proximity which they find in the solid state. This was first explained by Weiss as due to a large internal magnetic field set up by these atoms in the solid state, and the most recent theories have attempted to account for the mechanism by which this field is created. Heisenberg ${ }^{3}$ deduces it from the resonance, (the "Austausch-

* National Research Fellow.

${ }^{1}$ S. J. Barnett, Phys. Rev. [2] 36, 789 (1930).

${ }^{2}$ W. Gerlach and O. Stern, Zeits. f. Physik 9, 349 (1922), etc.

${ }^{3}$ W. Heisenberg, Zeits. f. Physik 49, 619 (1928). 
phänomene"), between the spinning electrons of neighboring atoms, while Bloch $^{4}$ believes the interaction takes place between the conduction electrons. It is therefore not surprising that ferromagnetism does not occur in liquids or gases where the separation of the atoms is greater. Further, as the orientations of the spinning electrons obeys a statistical energy distribution, the complicated nature of most ferromagnetic phenomena is accounted for, as well as the difficulty of using such measurements to work back to the mechanism of the individual atoms or electrons. Similar complications arise on the theoretical side, of a mathematical nature, and simplifying assumptions must be introduced, so that only predictions of a general character can be made.

In an attempt to simplify conditions it was thought interesting to observe the magnetic properties of ferromagnetic atoms in a state of isolation from one another intermediate between that of the pure solid metal and that of the Gerlach and Stern experiment. This suggested investigating alloys of a ferromagnetic with a non-ferromagnetic metal. By increasing the percentage of the latter the atoms of the former could be further and further isolated from each other and the corresponding effect on the magnetic properties noted. The present work is a continuation of that on the same subject previously reported by the author. ${ }^{5}$

\section{Experimental Method}

Several alloys of iron, nickel, or cobalt with other metals have been investigated. In nearly every case a small percentage of the non-magnetic element was sufficient to prevent ferromagnetism. For example, Lewkonja ${ }_{,}^{6}$ has tested alloys of $\mathrm{Co}$ with $\mathrm{Sn}, \mathrm{Sb}, \mathrm{Pb}, \mathrm{Bi}, \mathrm{Zn}, \mathrm{Cd}, \mathrm{Cr}$ and $\mathrm{Si}$ and Tammann, ${ }^{7}$ those of $\mathrm{Fe}, \mathrm{Ni}$ or $\mathrm{Co}$ with $\mathrm{Sn}, \mathrm{Sb}, \mathrm{Pb}, \mathrm{Bi}, \mathrm{Zn}, \mathrm{Si}, \mathrm{Al}$ and $\mathrm{Mg}$. In many cases, such as the $\mathrm{Ni}-\mathrm{Cr}$ series, ${ }^{8}$ the Curie point falls more and more rapidly to absolute zero as a few percent of, say, $\mathrm{Cr}$ is added. For $\mathrm{Ni}-\mathrm{Mn}^{9}$ it decreases steadily to $0^{\circ} \mathrm{C}$ for 25 percent $\mathrm{Mn}$. But in all these cases it is doubtful whether the two metals concerned form a continuous series of solid solutions, such as would be necessary for the purpose of this problem in order that the ferromagnetic atoms could be gradually further isolated. For the $\mathrm{Ni}-\mathrm{Cu}$ series, ${ }^{10}$ however, the Curie point reaches $-230^{\circ} \mathrm{C}$ for 65 percent $\mathrm{Cu}$, and it is believed that these two metals are mutually soluble except for a small range around 50 percent $\mathrm{Cu}$. But in the present work, alloys of cobalt have been selected in which the cobalt content can be made only a few percent without complete loss of ferromagnetism; these alloys belong to the Pt-Co and Pd-Co series. Although the diagram of thermal equilibrium has not been obtained for either of these series, Carter has investigated many high platinum alloys and believes platinum and cobalt to form an isomorphous series of solid solutions. As previous-

${ }^{4}$ F. Bloch, Zeits. f. Physik 57, 545 (1929).

${ }^{5}$ F. W. Constant, Phys. Rev. [2] 34, 1217 (1929).

${ }^{6}$ K. Lewkonja, Zeits. f. anorg. Chem. 59, 293 (1908).

${ }^{7}$ G. Tammann, Zeits. f. phys. Chem. 65, 73 (1909).

${ }^{8}$ C. Sadron, Comptes Rendus 190, 1339 (1930).

${ }^{9}$ S. Kaya and A. Kussmann, Naturwissenschaften 17, 995 (1929).

${ }^{10}$ R. Gans and A. Fonseca, Ann. d. Physik [4] 61, 742 (1920). 
ly reported, ${ }^{11}$ a microscopic investigation was made on each alloy used, confirming Carter's conclusions. Similar results were obtained for the Pd-Co alloys.

The first set of alloys investigated was the following, kindly supplied in the form of 30 mil wires by the Bell Telephone Laboratories:

$$
\begin{array}{r}
10 \% \mathrm{Co}-90 \% \mathrm{Pt} \\
5 \% \mathrm{Co}-95 \% \mathrm{Pt} \\
10 \% \mathrm{Ni}-90 \% \mathrm{Pt} \\
5 \% \mathrm{Ni}-95 \% \mathrm{Pt}
\end{array}
$$

The magnetic properties of these alloys have already been reported. The last two were not ferromagnetic, and the results obtained from the first two will be summarized below.

A second set of alloys was prepared, in the form of 10 mil wires whose compositions by weight were the following:

$$
\begin{gathered}
3 \% \mathrm{Co}-97 \% \mathrm{Pt} \\
1.5 \% \mathrm{Co}-98.5 \% \mathrm{Pt} \\
10 \% \mathrm{Co}-90 \% \mathrm{Pd} \\
5 \% \mathrm{Co}-95 \% \mathrm{Pd}
\end{gathered}
$$

The first two alloys served as a continuation of the Pt-Co series, while in the last two palladium was substituted for platinum so that by comparison with the Pt-Co series the contribution to the magnetic behavior of the cobalt atoms alone might be more truly ascertained. The experimental method was the same as that used with the first set of alloys, the magnetic measurements being made by the ballistic method, with two identical rings connected differentially. Measurements were taken on (1) the variation of the magnetization, $I$, (magnetic moment per unit volume), with temperature, and the Curie point, (2) hysteresis loops between various maximum values of the applied magnetic force, $H$, and at different temperatures, and (3) the initial curve of magnetization for various fixed temperatures. For the higher temperatures, $\left(50^{\circ} \mathrm{C}\right.$ to $\left.350^{\circ} \mathrm{C}\right)$, turpentine or paraffin baths were used, while for lower temperatures the specimens were placed in a Dewar flask containing liquid air or a bath of ether cooled by liquid air. Temperatures were measured by copper-constantan thermocouples.

The alloys were first tested in the hard-drawn state, when a microscopic examination showed the individual crystals greatly distorted and elongated; the wires were then annealed for three hours in a vacuum at about $1050^{\circ} \mathrm{C}$, and afterwards allowed to cool gradually. The furnace was a small electric one, built by A. Goetz, consisting of two concentric cylindrical crucibles with between them the heating element, a slightly conical helix of tungsten wire which served as a thread in which the inner crucible could be tightly screwed. After annealing the wires appeared microscopically to be composed of much larger crystals forming a polygonal pattern, characteristic of solid solutions.

${ }^{11}$ F. W. Constant, Phys. Rev. [2] 35, 116 (1930). 


\section{Results ANd Discussion}

The results for all the alloys in both the hard-drawn and annealed states are summarized in Tables I, II, and III.

TABle I. Curie Point.

\begin{tabular}{l|rrr|rc}
\hline \multirow{2}{*}{ Alloy } & \multicolumn{3}{c|}{ \% Cobalt } & \multicolumn{2}{c}{ Curie Point, ${ }^{\circ}$ Kelvin } \\
\cline { 2 - 6 } & by Wt. & by Vol. & Atomic & Hard-drawn & Annealed \\
\hline $90 \% \mathrm{Pt}$ & 10.0 & 21.4 & 26.9 & 578 & 522 \\
$95 \% \mathrm{Pt}$ & 5.0 & 11.4 & 14.9 & 322 & 322 \\
$97 \% \mathrm{Pt}$ & 3.0 & 7.1 & 9.3 & 193 & 179.5 \\
$98.5 \% \mathrm{Pt}$ & 1.5 & 3.7 & 4.8 & 57.5 \\
$90 \% \mathrm{Pd}$ & 10.0 & 13.4 & 16.8 & 511 & 462 \\
$95 \% \mathrm{Pd}$ & 5.0 & 6.8 & 8.7 & 359 & 301 \\
\hline \hline
\end{tabular}

In Table I are given the percentages of cobalt in each alloy by weight, by volume and from atomic considerations. The Curie points follow. For both series of alloys, in passing from 100 percent $\mathrm{Co}$, with Curie point $=1388^{\circ} \mathrm{K}$ to 0

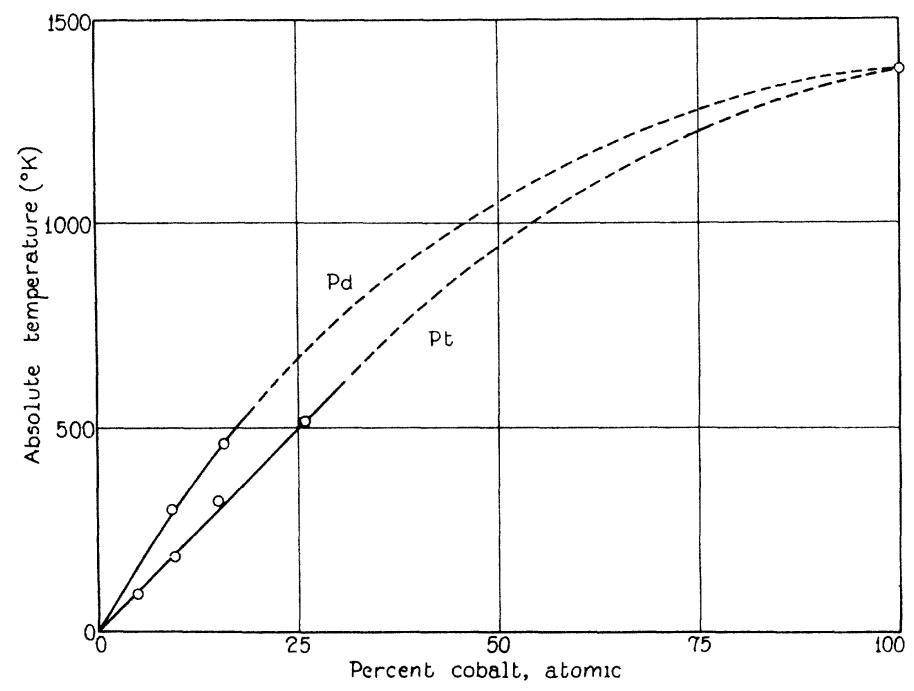

Fig. 1. Curie point as a function of the atomic percentage of cobalt.

percent $\mathrm{Co}$, the Curie point decreases more slowly at first and then falls approximately linearly with the atomic percentage of cobalt, approaching absolute zero for 0 percent Co. This is shown in Fig. 1. In the magnetization $-v s$. - temperature curves the magnetization at first increased with rising temperature, later falling to zero at the Curie point. This initial rise was explained as due to lack of saturation for the values of $H$ that could be applied.

Table II gives the characteristics of various hysteresis loops, the coercive force, $H_{c}$, and the remanent magnetization $I_{r}$, where $H_{\max }$ is the limiting value of $H$ for the loop. 
TABLE II. Coercive force and remanent magnetization.- $190^{\circ} \mathrm{C}$.

\begin{tabular}{lc|ccc|cc}
\hline \hline Alloy & $H_{\max }$ & Hard & $H_{c}$ & Annealed & Hard & $\begin{array}{c}I_{r} \\
\text { Annealed }\end{array}$ \\
\hline $90 \% \mathrm{Pt}$ & 260 & - & 30 & - & 189 \\
$95 \% \mathrm{Pt}$ & 260 & 90 & 18 & 200 & 107 \\
$97 \% \mathrm{Pt}$ & 260 & 80 & 14 & 73 & 53 \\
$98.5 \% \mathrm{Pt}$ & 260 & 30 & 0 & 1 & 142 \\
$90 \% \mathrm{Pd}$ & 260 & 120 & 110 & 210 & 167 \\
$90 \% \mathrm{Pd}$ & 650 & 420 & 50 & 88 & - & 83 \\
$95 \% \mathrm{Pd}$ & 260 & 410 & 110 & 145 & 108 \\
$95 \% \mathrm{Pd}$ & 650 & & & & \\
\hline \hline
\end{tabular}

The effect of annealing is seen to be to lower the Curie point and to decrease the magnetic hardness and hysteresis; the mechanical hardness was simultaneously decreased. This intimate connection between magnetic and mechanical hardness is in agreement with McKeehan' $\mathrm{s}^{12}$ theory of magnetostriction. O.v. Auwers and Sizoo ${ }^{13}$ have found that increasing the grain size by annealing produces just such results as these.

Decreasing the cobalt percentage always lowers the Curie point, and decreases $H_{c}$ and $I_{r}$. As the hysteresis decreases the loops become less upright and more inclined, and for the 98.5 percent alloy, annealed, no hysteresis was observed. This is in complete accord with the recent resonance theories of ferromagnetism, for if residual magnetism and hysteresis are explained as due to interaction between neighboring spinning electrons this interaction must diminish and the Curie point fall as these electrons become less dense. Comparison between the platinum and the palladium alloys shows similar results for both series except that $H_{c}$ is greater for the latter. The $\mathrm{Pt}$ or $\mathrm{Pd}$ atoms therefore seem to influence the hardness only and the ferromagnetism appears to be attributable to the cobalt atoms. It is of course possible that both $\mathrm{Pt}$ and $\mathrm{Pd}$ atoms contribute to the ferromagnetism in the same manner and degree but this seems less likely.

TABLE III. Maximum values of $I$ and the magnetic moment per cobalt atom.

\begin{tabular}{lccccc}
\hline \hline & Hard & $I_{\max }$ & Annealed & \multicolumn{1}{c}{$\begin{array}{l}I_{0, \infty} \\
\text { Annealed }\end{array}$} & $\sigma_{a} \times 10^{-20}$ \\
\hline $90 \% \mathrm{Pt}$ & 330 & 364 & 392 & 2.11 \\
$95 \% \mathrm{Pt}$ & 230 & 254 & 290 & 2.83 \\
$97 \% \mathrm{Pt}$ & 105 & 126 & 132 & 2.09 \\
$98.5 \% \mathrm{Pt}$ & 8 & 8 & 9 & 0.30 \\
$90 \% \mathrm{Pd}$ & 223 & 318 & 356 & 2.96 \\
$95 \% \mathrm{Pd}$ & 193 & 216 & \multirow{2}{*}{ 1 Bohr magneton $=0.92 \times 10^{-20}$} \\
\hline
\end{tabular}

In Table III the second and third columns give the greatest values of $I$ measured for each alloy. In most cases the greatest values of $H$ which could be applied, namely, 600 to 1000 gauss, were not sufficient to produce saturation, but the approach toward saturation followed closely, especially in the case of the palladium alloys, the formula given by Weiss and Forrer, ${ }^{14}$

${ }^{12}$ L. W. McKeehan, Phys. Rev. [2] 26, 274 (1925).

${ }^{13}$ O. v. Auwers and G. J. Sizoo, Zeits. f. Physik 60, 576 (1930).

${ }^{14}$ P. Weiss and R. Forrer, Ann. d. Physique [10] 12, 279 (1929). 


$$
I_{H}=I_{\infty}\left(1-\frac{a}{H}\right)
$$

where $I_{H}$ is the intensity of magnetization for a magnetic force $H, I_{\infty}$ the magnetization at saturation, $(H=\infty)$, and $a$ a constant for a given alloy and temperature. Table IV shows how closely the values of $I$ observed for the 10 percent Pd alloy fit those calculated from the formula

$$
I_{H}=356\left(1-\frac{131.4}{H}\right)
$$

TABLE IV. Approach to saturation for $90 \%$ Pd-10\% Co alloy.

\begin{tabular}{ccc}
\hline$H$ & $I$ calc. & I obs. \\
\hline 270 & 182 & 190 \\
361 & 226 & 225 \\
450 & 252 & 252 \\
564 & 273 & 272 \\
700 & 289 & 289 \\
767 & 295 & 295 \\
903 & 304 & 304 \\
$\infty$ & 356 & - \\
\hline \hline
\end{tabular}

In this way values of $I_{\infty}$ were computed at two different temperatures, that of liquid air and that of solid $\mathrm{CO}_{2}$ and alcohol. They were very nearly the same; if not, the value of $I_{\infty}$ at absolute zero, $I_{\infty, 0}$ could be calculated by the Weiss ${ }^{14}$ formula for temperature dependence,

$$
I_{\infty, T}=I_{\infty, 0}\left(1-A T^{2}\right)
$$

where $A$ is a constant and $T$ the absolute temperature. Recently Bloch ${ }^{15}$ has obtained a closely similar formula from his theory of ferromagnetism; namely,

$$
I_{\infty, T}=I_{\infty, 0}\left[1-\left(\frac{T}{\theta}\right)^{3 / 2}\right]
$$

where $\theta \doteq T_{c}$, the Curie point. In agreement with this formula, $I_{\infty, T}$ showed greater dependence on $T$ with those alloys whose Curie points were lowest. The fourth column of Table III gives the values computed for $I_{\infty, 0}$ and the fifth these values divided by the number of cobalt atoms per cc, i.e. the magnetic moment per cobalt atom, $\sigma_{a}$, assuming the magnetization is entirely due to the cobalt. For pure cobalt $\sigma_{a}$ is only about $1.6 \times 10^{-20}$ so that as the cobalt content decreases to 5 percent $\sigma_{a}$ increases, while for still further dilution it appears to decrease. This might be explained on Bloch's theory, according to which the spinning electrons between which resonance occurs are free conduction ones rather than associated with definite atoms. This electron gas can show ferromagnetism only if it is not completely degenerate so that its electrostatic energy may exceed its zero-point energy, (on the Sommerfeld theory). If decreasing the cobalt content in these alloys decreases the density of these free electrons the zero-point energy would decrease and this

${ }^{15}$ F. Bloch, Zeits. f. Physik 61, 206 (1930). 
might account for the increase in magnetic moment. At the same time, due to the lessening of the electrostatic energy a point would finally be reached where ferromagnetism would no longer be possible.

The theory of Bloch was prompted by the work of Dorfman, Jaanus and Kikoin ${ }^{16}$ on the change in atomic specific heat at the Curie point. Stoner ${ }^{17}$ has recently reported an important change in their results which alters the conclusion drawn from them that ferromagnetism is due to conduction electrons. But it is hard to distinguish between conduction and orbital electrons, and the peculiar electron arrangements in the $\mathrm{Fe}, \mathrm{Ni}$ and $\mathrm{Co}$ atoms would seem to be the most likely source of the ferromagnetism of these metals. It is probable that greater values of $\sigma_{a}$ would have been obtained with the 3 percent $\mathrm{Co}$ and 1.5 percent $\mathrm{Co}$ if the Curie points of these alloys had not been so low that measurements could only be taken a little below them.

In conclusion the following suggestions, along the lines of McKeehan's ${ }^{18}$ theory of ferromagnetism, are offered. Ferromagnetism occurs in certain metals only because in these metals the electrons are so arranged that resonance arises and the spins are oriented. Magnetic hardness arises because a change in the magnetization, or distribution of the spins, produces atomic magnetostriction, and hence gross magnetostriction unless the atomic magnetostriction of some of the atoms is of opposite sign so that compensation occurs. Such mechanical effects must be considered as partly influencing the degree of magnetization possible. As the percentage of non-magnetic or nonmagnetostrictive atoms increases, the atomic magnetostriction per cobalt atom can increase for a given gross magnetostriction. Assuming the magnetostriction of these alloys to be the same as for cobalt this would mean a greater observable magnetic moment per cobalt atom, as each cobalt atom in the alloy would be more free, with increasing dilution, to take up its state of greatest possible magnetization, than in pure cobalt. However, too great dilution of the cobalt would prevent ferromagnetism from occurring.

The writer wishes to thank the National Research Council for its support, and Professor R. A. Millikan for his kind help and for the facilities of the Norman Bridge Laboratory.

${ }^{16} \mathrm{~J}$. Dorfman, R. Jaanus and I. Kikoin, Zeits. f. Physik 54, 277, 289 (1929).

${ }^{17}$ E. Stoner, Nature 125, 973 (1930).

${ }^{18} \mathrm{~L}$. W. McKeehan, reference 12. 